Article

\title{
Novel Approach to Introduce Alkyl Chains into PEDOT:PSS and Its Effect on the Performance as a Flexible Electrode
}

\author{
In-Seong Hwang ${ }^{1}$, Chul-Woo Park ${ }^{1}$, Hye-In Kang ${ }^{1}$, Sung-yoon Joe ${ }^{2}$, Na-Young Pak ${ }^{3}$ and Dae-won Chung ${ }^{1, *}$ \\ 1 Department of Polymer Engineering, College of Engineering, The University of Suwon, \\ Hwaseong-si 18323, Korea; spbabois@suwon.ac.kr (I.-S.H.); 13039019@suwon.ac.kr (C.-W.P.); \\ gpdls2159@suwon.ac.kr (H.-I.K.) \\ 2 Center of Advanced Materials Analysis, The University of Suwon, Hwaseong-si 18323, Korea; \\ sungyoon.joe@suwon.ac.kr \\ 3 EverChemTech Co., Ltd., 38, Cheongwonsandan 7-gil, Mado-myeon, Hwaseong-si 18323, Korea; \\ pny@suwon.ac.kr \\ * Correspondence: dwchung@suwon.ac.kr; Tel.: +81-312-202-156
}

Citation: Hwang, I.-S.; Park, C.-W.; Kang, H.-I.; Joe, S.-y.; Pak, N.-Y.; Chung, D.-w. Novel Approach to Introduce Alkyl Chains into PEDOT:PSS and Its Effect on the Performance as a Flexible Electrode. Appl. Sci. 2021, 11, 6605. https:// doi.org/10.3390/app11146605

Academic Editor: Junseop Lee

Received: 30 June 2021

Accepted: 16 July 2021

Published: 18 July 2021

Publisher's Note: MDPI stays neutral with regard to jurisdictional claims in published maps and institutional affiliations.

Copyright: (c) 2021 by the authors. Licensee MDPI, Basel, Switzerland. This article is an open access article distributed under the terms and conditions of the Creative Commons Attribution (CC BY) license (https:// creativecommons.org/licenses/by/ $4.0 /)$.

\begin{abstract}
We here report a synthetic route to introduce alky chains into poly (3,4-ethylenedioxythiophene):poly (4-styrenesulfonate) (PEDOT:PSS) by the reaction with epoxyalkanes. The reaction was analyzed by FT-IR, TGA, and XPS studies, and the conductivities of derivatives were discussed as a function of the length of alkyl chains. PEDOT:PSS-C6, which is the product from a reaction with epoxyhexane, was well dispersed in methanol and transparent films from this dispersion were successfully prepared. PEDOT:PSS-C6 film showed an increase in hydrophobicity, resulting in enhanced water resistance compared to pristine PEDOT:PSS film, and a morphological study of the film exhibited clear phase separation similar to PEDOT:PSS doped by DMSO. We also observed an improvement in the conductivity and flexibility of PEDOT:PSS-C6 film compared to those of pristine PEDOT:PSS film. This study proposes a promising method to introduce alky chains into PEDOT:PSS and to develop a flexible electrode applicable to an environment where contact with water is unavoidable.
\end{abstract}

Keywords: PEDOT:PSS; epoxyalkanes; phase separation; water resistance; flexibility

\section{Introduction}

Poly (3,4-ethylenedioxythiophene) doped with poly (styrenesulfonate), PEDOT:PSS, is a transparent conductive polymer consisting of oligomeric PEDOT and polymeric PSS. Due to its unique combination of conductivity, transparency, ductility, and ease of processing, PEDOT:PSS has been benchmarked on various applications such as antistatic layers, electroluminescent devices, hole injection layers in organic light emitting diodes, charge transfer layers on biomedical electrodes, and wearable electrode [1-5]. It can also be used as a replacement for transparent conductors such as indium tin oxide (ITO) and is widely used in applications where the underlying substrate is flexible [6].

The PSS in the complex has two functions [7]. One is to act as dopants that stabilize cations in the main chain of PEDOT. The other is to disperse the PEDOT segments in the aqueous medium, and renders PEDOT:PSS an environmentally friendly polymer. However, hydrophilicity of PEDOT:PSS prevents its widespread application. For example, when PEDOT:PSS is employed as a material for the hole injection layer of an organic lightemitting device, indium is extracted from the electrode layer (ITO) due to the interaction of the sulfonic acid group of the PSS with water, and eventually shortening the lifespan of the organic light emitting device [8,9].

Therefore, a method of synthesizing PEDOT capable of dispersing in organic solvents by using a fluorine-based compound containing sulfonic acid [10] or an ionic liquid polymer $[11,12]$ as dopants instead of PSS has been under investigation. However, as long as 
the sulfonic acid-based dopant is used, the remaining sulfonic acid that is not engaged in doping with PEDOT chain eventually causes a problem of extracting and transferring indium through interaction with water.

In addition, various attempts have been made to stabilize PEDOT:PSS in the aqueous system. Berezhetska et al. used epoxy-containing silane coupling agents and successfully prevented delamination of PEDOT:PSS films in an aqueous environment [13]. Hakansson et al. reported that the epoxy group of the aforementioned silane coupling agents reacts with sulfonic acid group of PSS and the silanol group couples with glass substrate, which encourages formation of more stabilized film in the aqueous phase [14]. This behavior was also employed in a bioelectrode for in vivo recording of brain activity $[15,16]$

In this study, we prepared a derivative by reacting PSS with an epoxy containing alkyl groups focused on the fact that intrinsic properties of PEDOT:PSS can be altered by reacting PSS with the epoxy group. We isolated this product and characterized its structure by FT-IR, TGA, and XPS. We found that a derivative with hexyl group was well dispersed in methanol, and film formation was made possible per se without an additional solvent, binder, or wetting agent. We also investigated the electrical and physical properties of the film. This work demonstrates for the first time that derivatives of PEDOT:PSS reacted with epoxyalkanes have great potential for a flexible and water-stable electrode.

\section{Materials and Methods}

\subsection{Materials}

PEDOT:PSS (Clevios ${ }^{\mathrm{TM}}$ P, Heraeus Holding GmbH, Hanau, Germany) was used without further purification. The solid content, viscosity, and weight ratio of PEDOT to PSS were $1.2 \%$, less than $150 \mathrm{mPa} \cdot \mathrm{s}$, and 1:2.5, respectively. 1,2-Epoxybutane, 1,2-epoxyhexane, 1,2-epoxyoctane, 1,2-epoxybdodecane and 1,2-epoxyhexadecane, 2-propanol (IPA, 99.5\%), dimethyl sulfoxide (DMSO, 99.0\%), and silver paste (>75\%) were purchased from Sigma-Aldrich.

XPS and FT-IR spectra were recorded on a K-Alpha instrument (Thermo Scientific), and from $\mathrm{KBr}$ pellets on a Perkin Elmer spectrometer, respectively. The TGA (TGA 4000, Perkin Elmer) was carried out under a nitrogen atmosphere (nitrogen flow rate: $20 \mathrm{~mL} / \mathrm{min}$ ) while the temperature was increased from room temperature to $800^{\circ} \mathrm{C}$ at a rate of $10^{\circ} \mathrm{C} / \mathrm{min}$. Resistance of the films was monitored using a four point probe (M4P-302, MSTECH) setup with a source measurement unit (Keithley 2400). The values were converted into sheet resistance by taking correction factors into account [17]. For films coated on a glass, thickness was also measured by a surface profilometer (Alpha Step, P-10 of KLA-Tencor), and conductivity was calculated by using the following equation:

$$
\sigma=1 / \text { Rs } \cdot \mathrm{t}
$$

where $\sigma$ is conductivity, Rs is surface resistance, and $t$ is thickness.

Surface resistances of the films coated on a flexible film (PET), which are over $10^{3} \Omega / \mathrm{sq}$, were measured using a surface resistance meter MODEL ST-4 (SIMCO Japan). A two point probe (289 True RMS Multimeter, Fluke) was employed to measure linear resistance of films for the bending test. Surface properties of the films were evaluated by measuring the contact angle of the water droplet by utilizing a drop shape analyzer (DSA30, Kruss $\mathrm{GmbH}$ ). Surface morphology was studied by atomic force microscopy (AFM) (XE-100 of Park Systems).

\subsection{Reaction of PEDOT:PSS with Epoxyalkanes}

The following is an example of the reaction using epoxyhexane, and reactions with other epoxyalkanes were similarly carried out.

Corresponding to 10 times equivalent to the free sulfonic acid of PEDOT:PSS, $1.65 \mathrm{~g}$ of epoxyhexane was added to $50.0 \mathrm{~g}$ of PEDOT:PSS solution and stirred for $4 \mathrm{~h}$ at room temperature. The reactants were added dropwise into $500 \mathrm{~mL}$ of isopropanol, and the precipitate was recovered by filtration on nylon membrane $(0.2 \mu \mathrm{m}$, Whatman) and successively washed with isopropanol to remove unreacted epoxyalkanes. We obtained $0.55 \mathrm{~g}$ 
of the product after carefully drying under vacuum at $35^{\circ} \mathrm{C}$ for $8 \mathrm{~h}$. For epoxyoctane, epoxydecane, and epoxydodecane, we dissolved these in $50 \mathrm{~g}$ of methanol before adding to PEDOT:PSS, and washed with acetone the products obtained by the reaction of epoxydodecane. The products were labeled as PEDOT:PSS-Cn, where Cn means carbon numbers of reacted epoxyalkanes. For comparison, we added $50 \mathrm{~g}$ of PEDOT:PSS dropwise into 500 $\mathrm{mL}$ of isopropanol and recovered PEDOT:PSS as a solid state by filtration and dryness in the same manner. Detailed reaction conditions are summarized in Table 1.

Table 1. Reaction conditions and conductivities of PEDOT:PSS derivative.

\begin{tabular}{|c|c|c|c|c|c|c|}
\hline \multirow[b]{2}{*}{ Code } & \multirow[b]{2}{*}{$\begin{array}{c}\text { Carbon } \\
\text { Numbers of } \\
\text { Epoxyalkanes }\end{array}$} & \multicolumn{4}{|c|}{ Reaction Conditions } & \multirow[b]{2}{*}{$\begin{array}{l}\text { Conductivity } \\
\left(\mathrm{S} \cdot \mathrm{m}^{-1}\right)\end{array}$} \\
\hline & & $\begin{array}{c}\text { Weight of } \\
\text { PEDOT:PSS } \\
\text { Dispersion }^{\text {a) }} \text { (g) }\end{array}$ & $\begin{array}{c}\text { Weight of } \\
\text { Epoxyalkanes (g) }\end{array}$ & $\begin{array}{l}\text { Washing } \\
\text { Solvent }\end{array}$ & $\begin{array}{c}\text { Weight of } \\
\text { Products } \\
\text { Obtained (g) }\end{array}$ & \\
\hline PEDOT:PSS & - & $50 \mathrm{~g}$ & - & IPA & 0.51 & $132 \pm 31$ \\
\hline PEDOT:PSS-C4 & 4 & $50 \mathrm{~g}$ & 1.19 & IPA & 0.46 & $350 \pm 57$ \\
\hline PEDOT:PSS-C6 & 6 & $50 \mathrm{~g}$ & 1.65 & IPA & 0.55 & $268 \pm 16$ \\
\hline PEDOT:PSS-C8 & 8 & $50 \mathrm{~g}$ & 2.11 & IPA & 0.62 & $114 \pm 15$ \\
\hline PEDOT:PSS-C12 & 12 & $50 \mathrm{~g}$ & 3.03 & IPA & 0.58 & $94 \pm 39$ \\
\hline PEDOT:PSS-C16 & 16 & $50 \mathrm{~g}$ & 3.96 & Acetone & 0.48 & $55 \pm 11$ \\
\hline
\end{tabular}

a) Solid content is $1.2 \mathrm{wt}^{\mathrm{m}}{ }^{\mathrm{b})}$ The average and standard deviation values were calculated from 3 different samples independently prepared.

Derivatives after vacuum drying were ground with the pestle in a mortar and $120 \mathrm{mg}$ of ground powder was placed in the pellet die, which is generally used for making $\mathrm{KBr}$ pellet for IR spectroscopy. The pellet die was placed in a hydraulic press and pressed at $8000-10,000 \mathrm{psi}$ for $1 \mathrm{~min}$. The surface resistance and the thickness of the pellet-type derivatives were measured with a four-point probe and vernier calipers (CD-15CP of Mitutoyo), respectively.

\subsection{Preparation of the Films}

For preparation of films on a flexible film (PET), $60 \mathrm{mg}$ of PEDOT:PSS-C6 was dispersed in $10 \mathrm{~g}$ of methanol under sonication for $8 \mathrm{~h}$. The resulting $0.6 \mathrm{wt} . \%$ dispersion was coated on the PET film using \#10 Mayer bar coater (wet film thickness was $22.9 \mu \mathrm{m}$ ), and dried at $120^{\circ} \mathrm{C}$ for $1 \mathrm{~min}$. We measured transmittance of the films at $550 \mathrm{~nm}$, and converted this into \% transmittance by dividing the transmittance of coated films by that of the PET film itself. Surface resistance of the film was measured at four different points in one film, and the average value and standard deviation were evaluated. For PEDOT:PSS film, we added $5 \mathrm{~g}$ of IPA into $5 \mathrm{~g}$ of aqueous PEDOT:PSS dispersion (1.2 wt.\%), and coated on PET with \#10 Mayer bar coater. A similar process was employed for the preparation of films on a glass by spin coating ( $3000 \mathrm{rpm}, 1 \mathrm{~min}$ ) after the glass was cleaned in ethanol, and was employed under $\mathrm{O}_{2}$ plasma treatment.

\subsection{Bending Tests for the PEDOT:PSS-C6 Films}

We evaluated the flexibility of the films using a bending test apparatus (STS-RT-5AXIS, Sciencetown, South Korea). The PEDOT:PSS and PEDOT:PSS-C6 films coated on a PET film were cut into a rectangular piece, $1.5 \mathrm{~cm} \times 15 \mathrm{~cm}$, and the silver paste was applied at $1 \mathrm{~cm}$ from both ends of the films in the vertical direction (i.e., the gap between the silver pastes was $13 \mathrm{~cm}$ ). The film was attached to the bending machine, which was adjusted to 3R (curvature radius of $3 \mathrm{~mm}$ ) with the PEDOT:PSS coated side facing down. Therefore, PEDOT:PSS was folded by a 'outfolding' manner, as shown in Figure 1. The silver paste was connected to a multimeter (289 Fluke View, Fluke, Everett, WA, USA) to measure changes in the electric resistance. Changes of the electric resistance were recorded and stored by a computer connected to the multimeter, and analyzed. The bending test was conducted up to 50,000 cycles under $120 \mathrm{~Hz}$. 


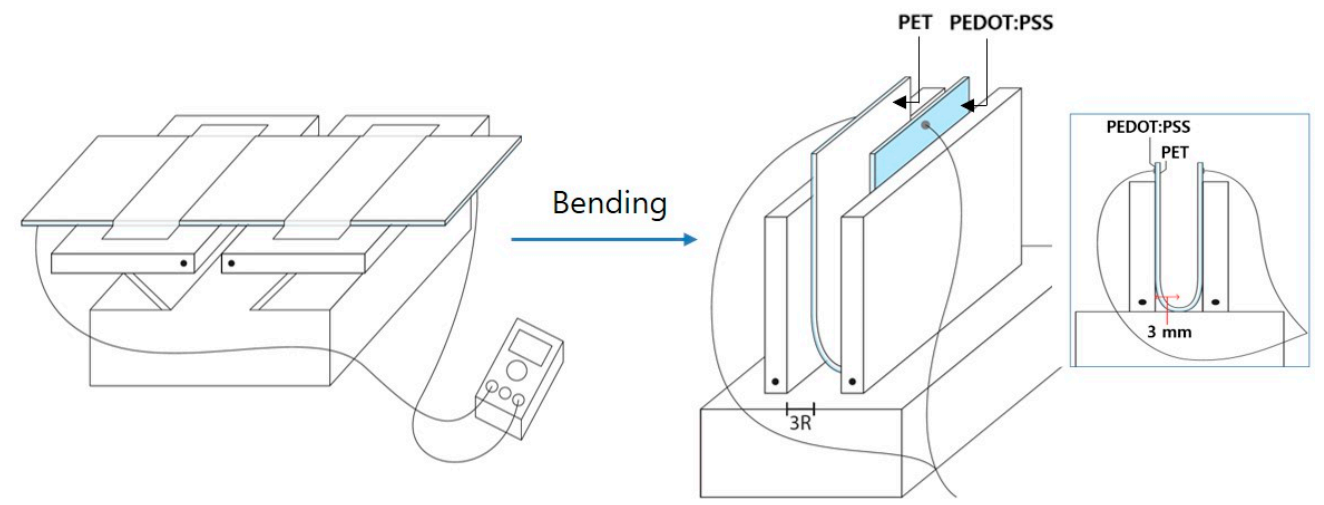

Figure 1. Schematic illustration of cyclic bending test. The insert illustrates a bended film viewed from the side.

\section{Results}

\subsection{Reaction of PEDOT:PSS with Epoxyalkanes}

The reaction between PEDOT:PSS and epoxyalkanes proceeded basically similar to a reaction between epoxy group and sulfonic acid. Considering the results of previous studies $[14,18]$, the reaction is speculated to proceed as shown in Figure 2. Since the $\mathrm{pH}$ of the PEDOT:PSS aqueous dispersion itself is about 2.0, it was carried out at room temperature without additional $\mathrm{pH}$ adjustment. For epoxyalkanes with $\mathrm{C} 8, \mathrm{C} 12$, and C16, which do not dissolve in water, we first dissolved each of them in methanol and then were added into an aqueous dispersion of PEDOT:PSS. As the reaction proceeded, the products settled into precipitation, and was easily recovered by filtration. To remove unreacted epoxyalkanes, the products were carefully washed with IPA, a good solvent for epoxyalkanes, and acetone for epoxydodecane. Table 1 shows the reaction conditions, the amount of reactants, and the conductivity values.

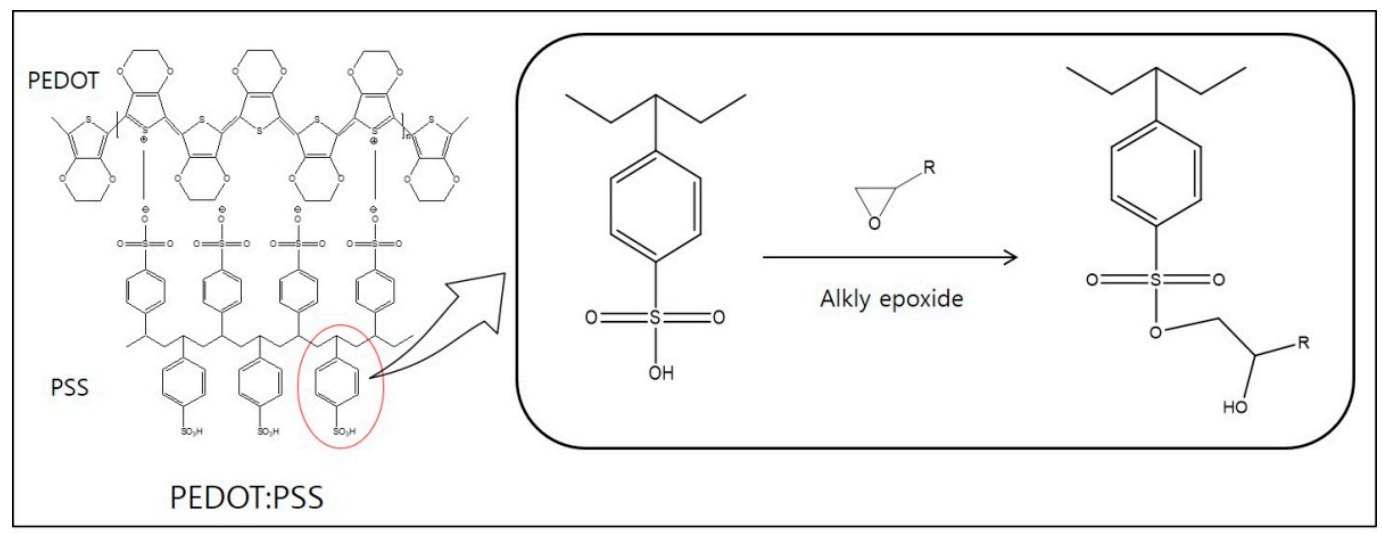

Figure 2. Suggested mechanism of the reaction of PEDOT:PSS with epoxyalkanes.

Epoxyalkanes were reacted in an amount corresponding to 10 times by equivalent to the free sulfuric acid of PSS in PEDOT:PSS, assuming that half of sulfonic acids is free and other half is engaged in doping with PEDOT chain [19]. Considering the solid content of the PEDOT:PSS aqueous dispersion used in this study is 1.2\% and the ratio of PEDOT and PSS is about 1:2.5 by weight as well, we used the following equation to obtain the equivalent values:

The equivalents of free sulfonic acid of PSS in $100 \mathrm{~g}$ of PEDOT:PSS dispersion

$$
\begin{aligned}
& =1 / 2 \times 100 \times 0.012 \times \text { wt. } \%(\text { PSS }) / \text { MW(PSS) } \\
& =0.0023 \text { equivalents }
\end{aligned}
$$


where wt.\% (PSS) is wt.\% of PSS in PEDOT:PSS (2.5/3.5 = 0.71), and MW (PSS) is the molecular weight of the repeating unit of PSS $\left(184 \mathrm{~g} \mathrm{~mol}^{-1}\right)$.

\subsection{Analysis of Chemical Structures}

We measured FT-IR to confirm the progress of the reaction. As shown in Figure 3a, in PEDOT:PSS the C-S vibration peaks due to thiophene structure appear at 830 and $972 \mathrm{~cm}^{-1}$ [20], and a small band due to the alkyl group appears at $2928 \mathrm{~cm}^{-1}$. However, in the case of the products, the absorption band of the alkyl group becomes larger. Basically, since the thiophene structure of PEDOT is not affected by the reaction, we can monitor the progress of the reaction by comparing the absorbances of the alkyl groups with those of the vibration peak caused by C-S. As indicated in Figure 3, the greater the length of the alkyl group, the larger it becomes. This result clearly indicates that the alkyl group was introduced by the reaction.

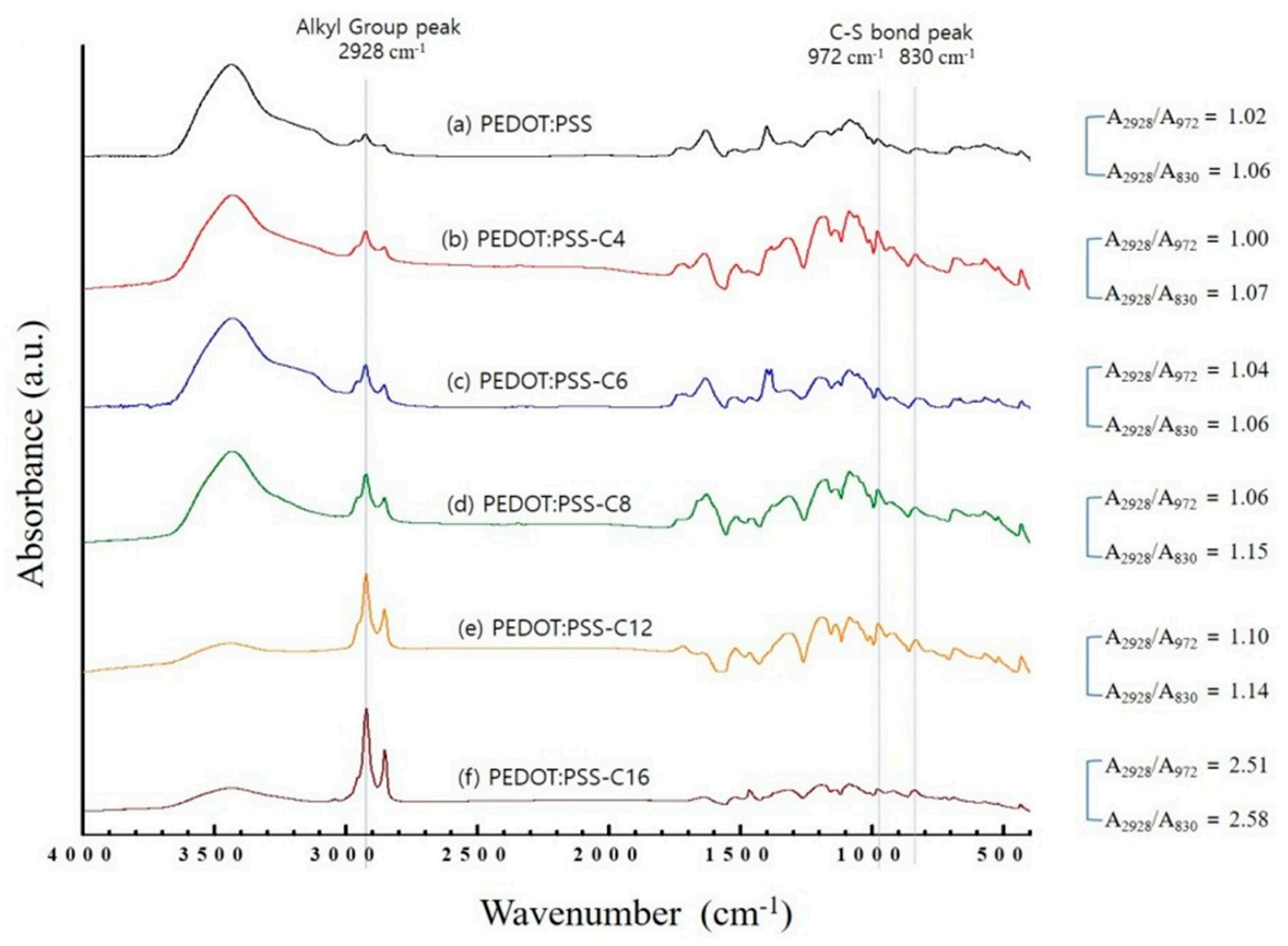

Figure 3. FT-IR spectra of PEDOT:PSS and derivatives.

Figure 4 shows the thermal properties for the pristine PEDOT:PSS and the products. As described in Figure 4a, all six samples show a slight mass loss at around $100{ }^{\circ} \mathrm{C}$, which is attributed to the loss of water and adsorbed solvent. The remaining weight at $800{ }^{\circ} \mathrm{C}$ decreases as the length of alkyl chain of epoxy alkane increases due to the fact that the alkyl groups easily decompose. As for pristine PEDOT:PSS, similar profile with published reports for a PEDOT:PSS film [21-23] was observed. In dTGA (Figure 4b), which can observe the change in the decomposition pattern, PEDOT:PSS showed second and third degradation temperature at around 350 and $400{ }^{\circ} \mathrm{C}$, which are due to the pyrolysis of sulfonic acid of PSS and polyethylene-type main chain of PSS, respectively [21,22]. Aromatics of PSS and thiophene groups of PEDOT are known to slowly decompose over $435^{\circ} \mathrm{C}$ [24]. On the other hand, when reacted with epoxyalkanes, decomposition of an alkyl group will appear at the third degradation temperature shown in PEDOT:PSS since the alkyl group has the same chemical structure as the polyethylene group. In fact, in products that reacted 
with epoxyalkanes, decomposition peaks around $400{ }^{\circ} \mathrm{C}$ are noticeable, and the peaks become more pronounced as the length of the alkyl group increases. These results strongly indicate that the alkyl groups are successfully introduced into PEDOT: PSS by the reaction with epoxyalkanes.
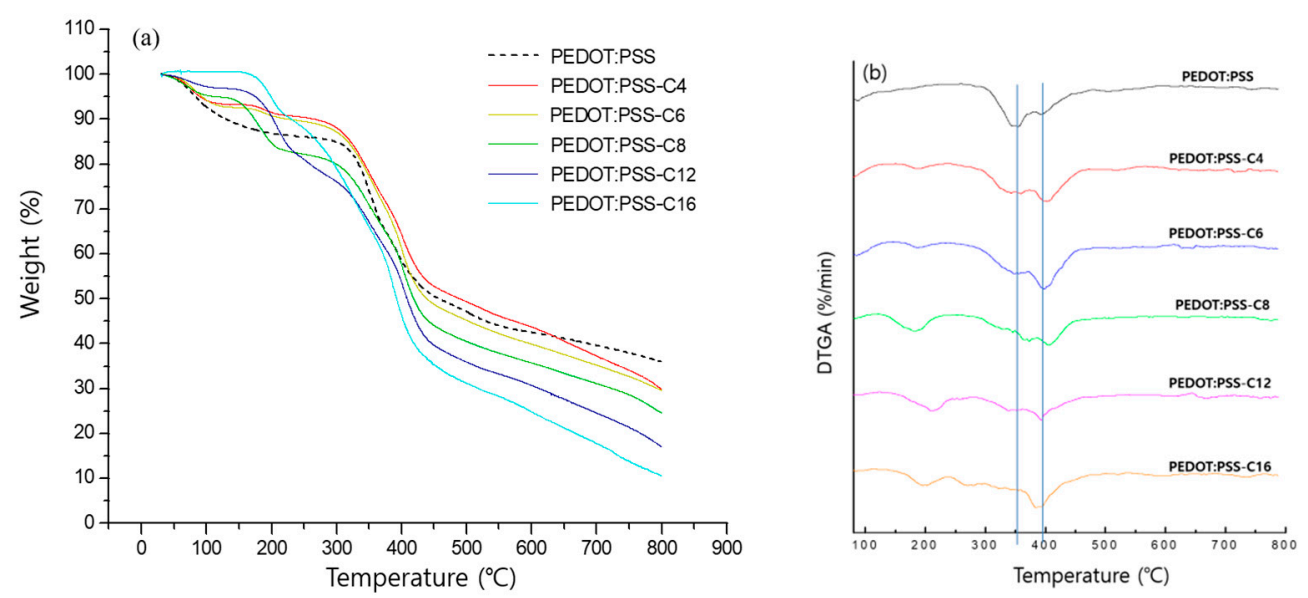

Figure 4. (a) TGA curves and (b) dTGA curves of PEDOT:PSS and derivatives.

We also studied the chemical structure of the products by XPS. The detailed deconvoluted patterns of $\mathrm{S}, \mathrm{C}$, and $\mathrm{O}$ atoms of three representative products are summarized in Figure 5. As can be seen in Figure $5 a, S_{2 p}$ core-level lines show two major peaks, which can be assigned to sulfur atom in PEDOT and PSS, respectively, and no significant change was observed by the reaction with epoxyalkanes. However, the $C_{1 s}$ spectra are strongly affected by the reaction with epoxyalkanes. For PEDOT:PSS, $\mathrm{C}_{1 \mathrm{~s}}$ signals can be divided into two major contributions with binding energies at about 284.2 and $285.9 \mathrm{eV}$, which are attributed to $\mathrm{C}-\mathrm{C} / \mathrm{C}=\mathrm{C}$ and $\mathrm{C}-\mathrm{O}$ species, respectively $[25,26]$. The product (PEDOT:PSSC4) that has reacted with epoxybutane also displays the same profile; however, the peaks corresponding to C-O turn out to be larger since the reaction with sulfonic acid in PSS and epoxide generate a new hydroxyl group. In fact, the area of $\mathrm{C}-\mathrm{O}$ relative to that of $\mathrm{C}-\mathrm{C} / \mathrm{C}=\mathrm{C}$ peak is 0.48 , whereas the relative areas is 0.34 for the pristine. The earlier study [18] on the reaction with PEDOT:PSS with epoxide compounds also demonstrated an increase in the peaks corresponding to C-O. On the other hand, for the reactions with epoxyalkanes with longer alkyl chains such as $\mathrm{C}-12$ and $\mathrm{C}-16$, the area of $\mathrm{C}-\mathrm{O}$ relative to that of $C-C / C=C$ peak shrank into 0.29 and 0.10 , respectively, since the $C-C$ peak increased. Reaction between PEDOT:PSS and epoxyalkanes was also confirmed from $\mathrm{O}_{1 \mathrm{~s}}$ signals. According to earlier reports $[18,27]$, the high binding energy contribution $(532.5 \mathrm{eV})$ in the pristine PEDOT:PSS is assigned to oxygen atoms in the ethylenedioxy segment in PEDOT, while peak at $530.8 \mathrm{eV}$ is assigned to $\mathrm{O}$ of the sulfonate groups in PSS. By reaction with epoxyalkanes, the peak due to sulfonate groups shifts toward high binding energies and the two peaks merge into a large signal around $532.2 \mathrm{eV}$. The merging of two peaks originating from $\mathrm{O}$ in PEDOT:PSS was also observed in the reaction with diepoxide compounds [18]. All these results strongly indicate that sulfonic acid of PSS reacts with epoxide of epoxyalkanes. 


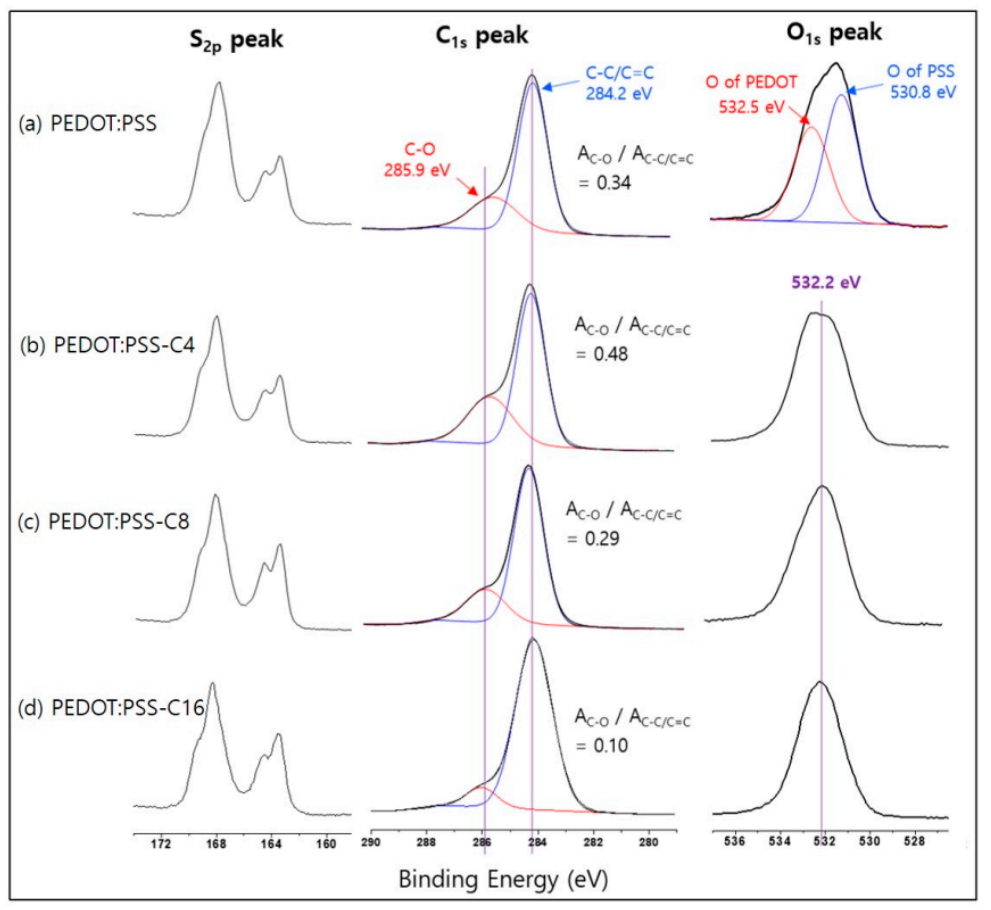

Figure 5. XPS spectra of PEDOT:PSS and derivatives.

In PEDOT:PSS, it is well known that half of the sulfuric acid of PSS act as dopant with PEDOT and the other half are present as free-acids, and the latter sulfuric acid can react with epoxide groups. In this study, we confirmed that it reacts with epoxyalkanes, as indicated in Figure 2.

\subsection{Properties of Epoxyalkane Derivatives}

The conductivity of PEDOT:PSS varies by the molar ratio of PEDOT to PSS and particle size. According to the review papers, which summarized electrical properties of several grades of commercials PEDOT:PSS, the conductivities of Clevious ${ }^{\mathrm{TM}} \mathrm{P}$, which is used in this study, are in the range of $20-100 \mathrm{~S} \cdot \mathrm{m}^{-1}$ [28] or about $100 \mathrm{~S} \cdot \mathrm{m}^{-1}$ [29]. In this study, the conductivity value of PEDOT:PSS was found to be $132 \mathrm{~S} \cdot \mathrm{m}^{-1}$, as noted in Table 1 . In the case of the derivatives, the greater the length of the reacted alkyl group, the lower the electrical conductivity, which is due to the fact that the amount of PEDOT responsible for conductivity in the entire molecule is relatively reduced as the chain length becomes longer. It is worth noting that the conductivities of PEDOT:PSS-C4 and -C6 are higher than those of pristine PEDOT:PSS. It is well known that the conductivity of PEDOT:PSS film significantly improves by the addition of organic solvents such as DMSO [30], ionic liquids [31], or surfactants [32], which is usually referred to as 'secondary doping'. The conductivity enhancement of PEDOT:PSS film through a post-treatment with organic solvents [33] or acidic compounds [34] has been investigated in many studies as well. All of these methods are well summarized in a review paper [35]. However, the conductivity of PEDOT:PSS-C4 and -C6 itself, without an additive or any further post-treatment, is higher than that of pristine PEDOT:PSS. To our knowledge, it is the first example.

All derivatives were not dispersed in water due to the absence of sulfonic acid groups of PSS, which mainly contribute to the hydrophilicity of PEDOT:PSS [7]. On the other hand, PEDOT:PSS-C6 was well dispersed in methanol at a concentration of $0.6 \mathrm{wt} . \%$, which made it possible for us to prepare a transparent film. By studying the properties of this film, we attempted to elucidate the principle of the exceptional conductivity increase. 


\subsection{Properties of PEDOT:PSS-C6 Films}

The films of PEDOT:PSS and PEDOT:PSS-C6 were prepared by bar-coating, and we also made films containing $2 \mathrm{wt} . \%$ DMSO as the second dopant. Table 2 displays surface resistance and transmittance of the films.

Table 2. Properties of PEDOT:PSS and PEDOT:PSS-C6 films and their doped films.

\begin{tabular}{|c|c|c|c|c|c|c|c|c|}
\hline & Code & DMSO & $\begin{array}{c}\text { Contact } \\
\text { Angle }\left({ }^{\circ}\right)\end{array}$ & $\begin{array}{c}\text { Surface } \\
\text { Energy } \\
(\mathrm{mN} / \mathrm{m})\end{array}$ & $\begin{array}{c}\text { Transmittance } \\
(\%)\end{array}$ & $\begin{array}{c}\text { Surface } \\
\text { Resistance }^{\text {a) }} \\
(10 \times \Omega / \text { sq })\end{array}$ & $\begin{array}{l}\text { Conductivity } \\
\text { b) }(\mathrm{S} / \mathrm{m})\end{array}$ & $\begin{array}{c}\text { Surface } \\
\text { Roughness. } \\
\text { RMS (nm) }\end{array}$ \\
\hline (I) & PEDOT:PSS & - & 49.9 & 52.4 & 97.5 & 6.2 & $155 \pm 59$ & 2.539 \\
\hline (II) & PEDOT:PSS & $2 w t \%$ & - & - & 98.2 & 3.1 & - & 3.207 \\
\hline (III) & PEDOT:PSS-C6 & - & 83.0 & 34.6 & 97.7 & 5.6 & $354 \pm 81$ & 5.141 \\
\hline (IV) & PEDOT:PSS-C6 & $2 w t \%$ & - & - & 97.5 & 3.1 & - & 5.757 \\
\hline
\end{tabular}

a) 5 different points of a film $(200 \mathrm{~mm} \times 250 \mathrm{~mm})$ were measured, and the average and standard deviation were calculated. ${ }^{\text {b) }}$ Conductivities were calculated from the surface resistance values and the thickness of the films prepared by the spin coating on the glass. The thickness was measured by alpha step. The average and standard deviation values were calculated from 3 different samples independently prepared.

That all of the films we prepared were found to be well wetted and transmittance also exhibited over $97 \%$ vindicates that they maintain their properties as transparent films. To exclude latent effect by dopants, we also measured contact angle to observe surface properties of films where we used two kinds of film containing no DMSO. Figure 6 displays the state of the films on which water droplets were placed and then allowed to stand for $9 \mathrm{~s}$. As can be seen, the contact angle shows a rapid decrease in PEDOT:PSS while there is hardly any change in contact angle in PEDOT:PSS-C6 films, implying improved water resistance. In addition, we measured contact angles for $9 \mathrm{~s}$ at an interval of one second and took an average from which we calculated surface energy values (Table 2). As can be expected from the photos, PEDOT:PSS-C6 film exhibited higher contact angle and surface energy compared to the pristine, and it is conceivable that introduction of alkyl groups contribute to an increase in hydrophobicity. This is consistent with the phenomenon that $\mathrm{SO}_{3} \mathrm{H}$ moiety of PSS disappears after reaction in PEDOT:PSS [14,18].

(a)

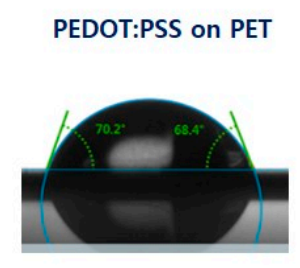

(b)

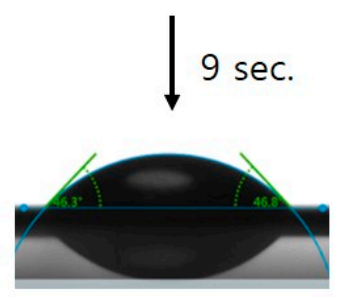

PEDOT:PSS-C6 on PET

(c)

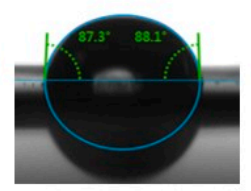

$9 \mathrm{sec}$.

(d)

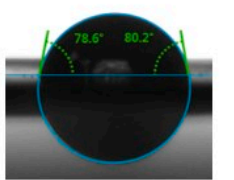

PEDOT:PSS on glass

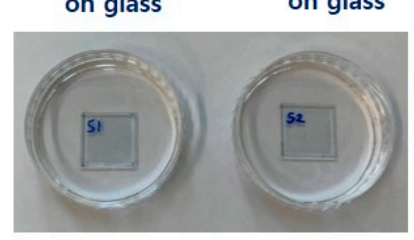

$20 \mathrm{hrs}$

(f)

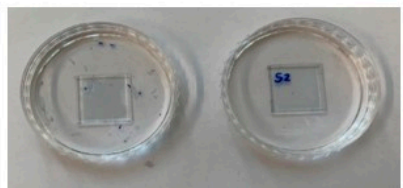

Figure 6. Image of contact angles formed by liquid water drops on the surface of (a) PEDOT:PSS film, (c) PEDOT:PSS-C6 film, and (b,d) after $9 \mathrm{~s}$ for each film. Photos of (e) PEDOT:PSS and PEDOT:PSS-C6 film immersed in water, and (f) after $20 \mathrm{~h}$.

When the films prepared by spin-coating with identical coating solution on the glass substrate are immersed in water and then $20 \mathrm{~h}$ is allowed to pass, the pristine film detached from the glass and shattered into pieces. In contrast, PEDOT:PSS-C6 film maintained its state, from which we can visually confirm enhanced water resistance. 
When comparing the surface resistance values of two kind of films without DMSO, PEDOT:PSS-C6 films (III) showed much lower surface resistance than the film (I) of PEDOT:PSS only, as described in Table 2. This is the same result we recorded in Table 1, confirming that the electrical property of PEDOT:PSS-C6 itself is more conspicuous than PEDOT:PSS. When DMSO was added as the second dopant, PEDOT:PSS (II) and PEDOT:PSSC6 (IV) films exhibit similar values. That is, in the case of PEDOT:PSS-C6, the electrical properties were superior to that of pristine, but the effect of the secondary doping was less. We used AFM to investigate the differences in morphology of these four films for the confined area of $2 \mu \mathrm{m} \times 2 \mu \mathrm{m}$, which is sufficiently large to provide simultaneously reliable analysis.

As seen in Figure 7a, where AFM images of four kinds of films are compared in the height mode, the surface of PEDOT:PSS film (I) without a dopant is very smooth, while there are grains for the doped film (II). The root-mean-square roughness (RMS), the most commonly evaluated parameter representing the surface roughness, is 2.539 and $3.257 \mathrm{~nm}$ for (I) and (II), respectively. There are many research activities on measuring the roughness of PEDOT:PSS films and their data exhibit similar values that we have observed. For example, PEDOT:PSS films without doping and doped with 2 wt.\% DMSO showed RMS values with 2.51 and $3.61 \mathrm{~nm}$, respectively [36]. In contrast, both PEDOT:PSS-C6 films (III) and (IV) show high RMS values (5.141 $\mathrm{nm}$ and $5.757 \mathrm{~nm}$, respectively) regardless of the doping process.

(a)
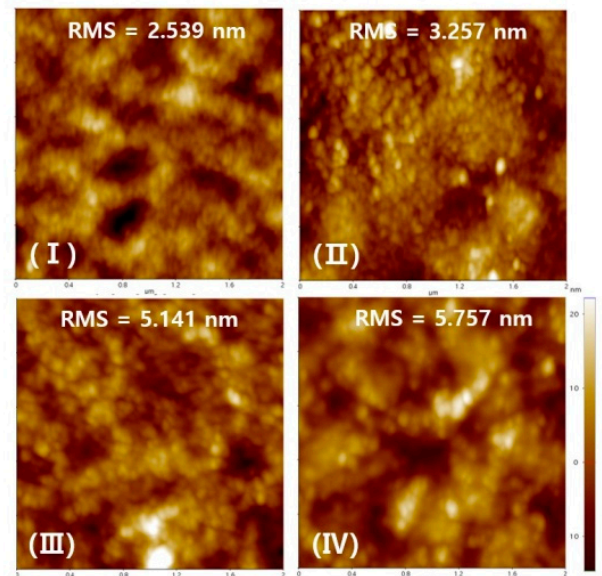
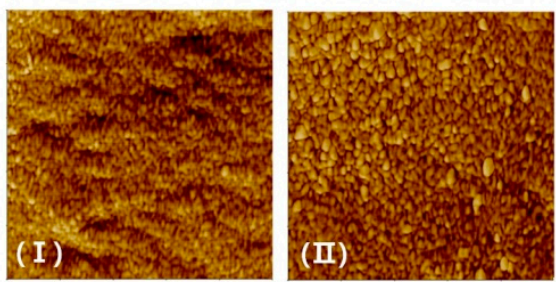

(b)
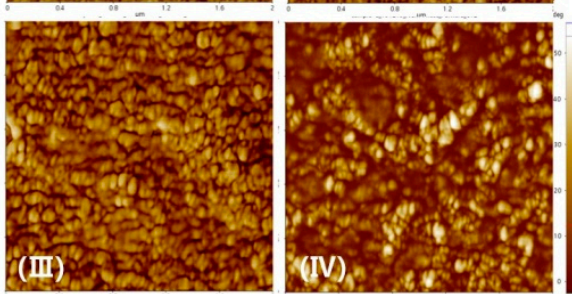

Figure 7. AFM image of (a) surface morphology and (b) phase shift of PEDOT:PSS films undoped (I), doped (II) with DMSO, PEDOT:PSS-C6 film undoped (III), and doped (IV) with DMSO.

Since Kim et al. reported that incorporation of polar and high boiling-point solvents such as DMSO contributes to the improvement in conductivity [30], various attempts have been made to further increase conductivity by adding different additives or post-treatment. All of these phenomena are called secondary doping, and this second doping process is speculated to be triggered by separation of PSS chains from PEDOT [35,37,38]. In recent studies [36] on the relationship between the effect of various secondary dopants and the morphology of PEDOT:PSS film, the phase separation phenomenon accompanying the enhancement of conductivity was analyzed more reliably in the phase-shift mode of AFM. In this study, we also observed phase-shift images (Figure $7 \mathrm{~b}$ ) of the same four films.

The PEDOT:PSS film (I) without doping shows small islands that are irregular in shape, and the doped film (II) displays globular grains with clear boundaries, which suggests the occurrence of phase separation. This result is similar to the observation reported by Donoval et al. [36]. On the other hand, PEDOT:PSS-C6 film (III) also shows a phaseseparated image with globular grains with clear boundaries, which is similar to that of doped film (II). No significant change is observed in the doped film (IV) of PEDOT:PSS-C6, which strongly indicates that the doping of PEDOT:PSS-C6 with DMSO does not affect the morphology or the conductivity of PEDOT:PSS-C6. 
In summary, the surface resistance of the undoped PEDOT:PSS-C6 film (III) was significantly lower than that of pristine PEDOT:PSS (I), and showed a value similar to that of the PEDOT:PSS film (II) doped with DMSO. The study on morphology of film (III) clearly showed phase separation similar to the film (II). Accordingly, in PEDOT:PSS-C6, it is understood that the introduction of an alkyl group into the sulfuric acid of the free PSS of PEDOT:PSS causes phase separation more clearly and leads to an improvement in conductivity.

\subsection{Flexible Properties of PEDOT:PSS-C6 Films}

Films of organic polymers such as PEDOT:PSS have intrinsic flexible properties. In fact, chemical vapor deposition graphene [39] and Ag nanowires [40] were rendered flexible by the overcoating of PEDOT:PSS. However, under extreme bending conditions the conductive network of PEDOT:PSS is known to be unretained. For example, the initial sheet resistance of the PEDOT:PSS film on PET or PI substrates increased by $48.3 \%$ or $54.4 \%$, respectively, after 1000 bending cycles in a curvature of 0 to 180 degrees [41].

Cyclic bending tests were conducted to examine the mechanical reliability of the films. Change in surface resistance as a function of the number of bending cycles at a fixed $3 \mathrm{~mm}$ bending curvature was monitored, as described schematically in Figure 1. Basically, the deviation was large in the results of each test, but a meaningful trend was confirmed from the average and standard deviation of the three independent experiments, as shown in Figure 8. In the earlier 'infolding' experiment [18], where PEDOT:PSS layer coated on PET substrate was folded inward, surface resistances were stable until 50,000 cycles of bending. However, in the 'outfolding' bending experiment, PEDOT:PSS displayed a large deviation in surface resistance, even in the initial stage of cycles, and after 100,000 cycles $\mathrm{R} /$ Ro values reached 1.2. Bending in an 'outfolding' manner induced direct stretching and a conductive network can be easily disconnected compared to that under 'infolding' bending. On the other hand, PEDOT:PSS-C6 films showed relatively stable resistances, which suggests that the conductive network structure of PEDOT:PSS was clearly stabilized. In general, alkyl chains are considered as a flexible group. In fact, it is reported that alkylsubstituted semiconductors played an important role in the stability of the flexible electronic devices [42]. In the same manner, we speculate that the hexyl chain of PEDOT:PSS-C6 gives the higher flexibility compared to PEDOT:PSS. We tentatively conclude that the introduction of the alkyl group into the free sulfonic acid group of PEDOT:PSS improves the flexibility and water resistance of PEDOT:PSS.

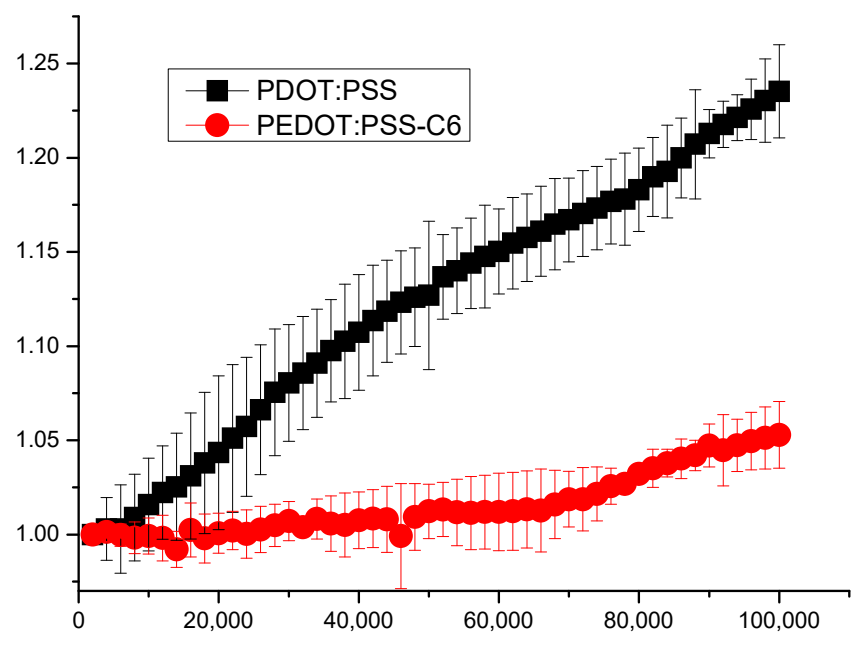

Figure 8. Surface resistance ratio (R/Ro) of PEDOT:PSS films as a function of bending cycles at a curvature radius of $3 \mathrm{~mm}$. 


\section{Conclusions}

We successfully prepared derivatives of PEDOT:PSS by the reaction with epoxyalkanes and the filtration of the reaction mixture on a conventional nylon filter. FT-IR, TGA, and XPS studies clearly demonstrate that alkyl chains are introduced to PEDOT:PSS by the reaction between the free sulfonic acid of PSS and epoxide group. In the case of derivatives, the greater the length of the reacted alkyl group, the lower the electrical conductivity; on the other hand, it is worth noting that the conductivities of PEDOT:PSS-C4 and -C6 are higher than those of pristine PEDOT:PSS.

Most of the derivatives were not dispersed in water or alcohol-based solvents; however, PEDOT:PSS-C6 was well dispersed in methanol at a concentration of $0.6 \mathrm{wt} . \%$. We succeeded in preparing a transparent film from this dispersion on a glass or a flexible substrate (PET). An increase in hydrophobicity and subsequence enhanced water resistance of PEDOT:PSS-C6 film were confirmed by measuring contact angle and visually observing the film in water, respectively. The surface roughness of PEDOT:PSS-C6 film was much higher than that of pristine PEDOT:PSS film, and nearly the same roughness as that of PEDOT:PSS film doped by DMSO. Further studies on morphology of PEDOT:PSS-C6 film clearly showed phase separation similar to the doped PEDOT:PSS film. When the flexibility of films was examined by cyclic bending tests, surface resistance of PEDOT:PSS-C6 was found to be more stable compared to that of PEDOT:PSS.

We tentatively conclude that, in PEDOT:PSS-C6, the introduction of the alkyl group into the sulfuric acid of the free PSS of PEDOT:PSS causes phase separation more distinctively and finally leads to an improvement in conductivity. Furthermore, the alky groups introduced into PEDOT:PSS induced the improvements of the flexibility and water resistance of PEDOT:PSS.

Author Contributions: Conceptualization, D.-w.C.; formal analysis, S.-y.J. and N.-Y.P.; methodology, I.-S.H., C.-W.P. and H.-I.K.; writing-original draft, I.-S.H., H.-I.K. and D.-w.C.; writing-review and editing, I.-S.H. and D.-w.C. All authors have read and agreed to the published version of the manuscript.

Funding: This research was funded by the research grant of the University of Suwon in 2019.

Conflicts of Interest: The authors declare no conflict of interest.

\section{References}

1. Groenendaal, L.; Jonas, F.; Freitag, D.; Pielartzik, H. Poly(3,4-ethylenedioxythiophene) and Its Derivatives: Past, Present, and Future. Adv. Mater. 2000, 12, 481-494. [CrossRef]

2. Asplund, M.; Nyberg, T.; Inganäs, O. Electroactive polymers for neural interfaces. Polym. Chem. 2010, 1, 1374-1391. [CrossRef]

3. Yoon, D.G.; Kang, M.G.; Kim, J.B.; Kang, K.T. Nozzle Printed-PEDOT:PSS for Organic Light Emitting Diodes with Various Dilution Rates of Ethanol. Appl. Sci. 2018, 8, 203. [CrossRef]

4. Zeng, W.; Shu, L.; Li, Q.; Chen, S.; Wang, F.; Tao, X. Fiber-based wearable electronics: A review of materials, fabrication, devices, and Applications. Adv. Mater. 2014, 26, 5310-5336. [CrossRef]

5. Yan, W.; Dong, C.; Xiang, Y.; Xiang, Y.; Jiang, S.; Leber, A.; Loke, G.; Xu, W.; Hou, C.; Zhou, S.; et al. Thermally drawn advanced functional fibers: New frontier of flexible electronics. Mater. Today 2020, 35, 168-194. [CrossRef]

6. Chen, S.; Song, L.; Tao, Z.; Shao, X.; Huang, Y.; Cui, Q.; Guo, X. Neutral-pH PEDOT:PSS as over-coating layer for stable silver nanowire flexible transparent conductive films. Org. Electron. 2014, 15, 3654-3659. [CrossRef]

7. Greczynski, G.; Kugler, T.; Salaneck, W.R. Characterization of the PEDOT-PSS system by means of X-ray and ultraviolet photoelectron spectroscopy. Thin Solid Film. 1999, 354, 129-135. [CrossRef]

8. De Jong, M.P.; van IJzendoorn, L.J.; De Voigt, M.J.A. Stability of the interface between indium-tin-oxide and poly (3,4ethylenedioxythiophene)/poly (styrene sulfonate) in polymer light-emitting diodes. Appl. Phys. Lett. 2000, 77, 2255-2257. [CrossRef]

9. Crispin, X.; Marciniak, S.; Osikowicz, W.; Zotti, G.; van der Gon, A.W.D.; Louwet, F.; Fahlman, M.; Groenendaal, L.; De Schryver, F.; Salaneck, W.R. Conductivity, Morphology, Interfacial Chemistry, and Stability of Poly(3,4-ethylene dioxythiophene)-Poly(styrene sulfonate): A Photoelectron Spectroscopy Study. Pol. Phys. 2003, 41, 2561-2583. [CrossRef]

10. Li, L.; Zhang, Y. Chemical modification of Nafion membrane with 3,4-ethylenedioxythiophene for direct methanol fuel cell application. J. Power Sources 2007, 175, 256-260. [CrossRef] 
11. Kim, T.Y.; Suh, M.W.; Kwon, S.J.; Lee, T.H.; Kim, J.E.; Lee, Y.J.; Kim, J.H.; Hong, M.P. Poly(3,4-ethylenedioxythiophene) Derived from Poly(ionic liquid) for the Use as Hole-Injecting Material in Organic Light-Emitting Diodes. Macromol. Rapid Commun. 2009, 30, 1477-1482. [CrossRef] [PubMed]

12. Marcilla, R.; Ochoteco, E.; Pozo-Gonzalo, C.; Grande, H.; Pomposo, J.A.; Mecerreyes, D. New Organic Dispersions of Conducting Polymers Using Polymeric Ionic Liquids as Stabilizers. Macromol. Rapid Commun. 2005, 26, 1122-1126. [CrossRef]

13. Berezhetska, O.; Liberelle, B.; De Crescenzo, G.; Cicoira, F. A simple approach for protein covalent grafting on conducting polymer films. J. Mater. Chem. B 2015, 3, 5087-5094. [CrossRef]

14. Hakansson, A.; Han, S.B.; Wang, S.H.; Lu, J.; Braun, S.; Fahlman, M.; Berggren, M.; Crispin, X.; Fabiano, S. Effect of (3glycidyloxypropyl) trimethoxysilane (GOPS) on the electrical properties of PEDOT:PSS films. J. Polym. Sci. Pol. Phys. 2017, 55, 814. [CrossRef]

15. Khodagholy, D.; Doublet, T.; Quilichini, P.; Gurfinkel, M.; Leleux, P.; Ghestem, A.; Ismailova, E.; Herve, T.; Sanaur, S.; Bernard, C.; et al. In vivo recordings of brain activity using organic transistors. Nat. Commun. 2013, 4, 1575. [CrossRef] [PubMed]

16. Khodagholy, D.; Doublet, T.; Gurfinkel, M.; Quilichini, P.; Ismailova, E.; Leleux, P.; Herve, T.; Sanaur, S.; Bernard, C.; Malliaras, G.G. Highly Conformable Conducting Polymer Electrodes for In Vivo Recordings. Adv. Healthc. Mater. 2011, 23, H268-H272. [CrossRef] [PubMed]

17. Perloff, D.S. Four-point sheet Resistance Correction Factors for Thin Rectangular samples. Solid-State Electron. 1977, 20, 681-687. [CrossRef]

18. Park, M.U.; Lee, M.L.; Chung, D.W. Model system of cross-linked PEDOT:PSS adaptable to an application for an electrode with enhanced water stability. Synth. Met. 2019, 258, 116195. [CrossRef]

19. Jonsson, S.K.M.; Birgerson, J.; Crispin, X.; Greczynski, G.; Osikowicz, W.; Denier van der Gon, A.W.; Salaneck, W.R.; Fahlman, M. The effects of solvents on the morphology and sheet resistance in poly (3,4-ethylenedioxythiophene)-polystyrenesulfonic acid (PEDOT-PSS) films. Synth. Met. 2003, 139, 1-10. [CrossRef]

20. Cho, W.; Wu, J.; Shim, B.S.; Kuan, W.F.; Mastroianni, S.E.; Young, W.S.; Kuo, C.C.; Epps, T.H.; Martin, D.C. Synthesis and characterization of bicontinuous cubic poly (3,4-ethylene dioxythiophene) gyroid (PEDOT GYR) gels. Chem. Phys. 2015, 17, 5115-5123. [CrossRef]

21. Ahmad, I.; McCarthy, J.E.; Baranov, A.; Gun'ko, Y.K. Development of Graphene Nano-Platelet Based Counter Electrodes for Solar Cells. Materials 2015, 8, 5953-5973. [CrossRef]

22. Friedel, B.; Keivanidis, P.E.; Brenner, T.J.K.; Abrusci, A.; McNeill, C.R.; Friend, R.H.; Greenham, N.C. Effects of Layer Thickness and Annealing of PEDOT:PSS Layers in Organic Photodetectors. Macromolecules 2009, 42, 6741-6747. [CrossRef]

23. Xu, Y.; Wang, Y.; Liang, J.; Huang, Y.; Ma, Y.; Wan, X.; Chen, Y. A hybrid material of graphene and poly (3,4-ethyldioxythiophene) with high conductivity, flexibility, and transparency. Nano Res. 2009, 2, 343-348. [CrossRef]

24. Lee, C.J.; Tsai, I.S. Preparation and Properties of PEDOT/PSS Conductive Polymer Blended with Graphene/PVDF. Adv. Mater. Res. 2013, 608, 1318-1326. [CrossRef]

25. Park, N.I.; Lee, S.B.; Lee, S.M.; Chung, D. Preparation and Characterization of PEDOT/PSS Hybrid with Graphene Derivative Wrapped by Water-soluble Polymer. Appl. Chem. Eng. 2014, 25, 581-585. [CrossRef]

26. Greczynski, G.; Kugler, T.; Keil, M.; Osikowicz, W.; Fahlman, M.; Salaneck, W.R. Photoelectron spectroscopy of thin films of PEDOT-PSS conjugated polymer blend: A mini-review and some new results. J. Electron Spectrosc. Relat. Phenom. 2001, $121,1-17$. [CrossRef]

27. Ahn, M.H.; Cho, E.S.; Kwon, S.J. Process Optimization of ITO Film on PC Substrate Deposited by In-line Sputtering Method for a Resistive-type Touch Panel. J. Korean Vac. Soc. 2009, 18, 440-446. [CrossRef]

28. Shi, H.; Liu, C.; Jiang, Q.; Xu, J. Effective Approaches to Improve the Electrical Conductivity of PEDOT:PSS: A Review. Adv. Electron. Mater. 2015, 1, 1500017. [CrossRef]

29. Kirchmeyer, S.; Reuter, K. Scientific importance, properties and growing applications of poly (3,4-ethylenedioxythiophene). J. Mater. Chem. 2005, 15, 2077-2088. [CrossRef]

30. Kim, J.Y.; Jung, J.H.; Lee, D.E.; Joo, J. Enhancement of electrical conductivity of poly (3,4-ethylenedioxythiophene)/poly (4-styrenesulfonate) by a change of solvents. Synth. Met. 2002, 126, 311-316. [CrossRef]

31. Badre, C.; Marquant, M.; Alsayed, A.M.; Hough, L.A. Highly Conductive Poly(3,4-ethylenedioxythiophene): Poly(styrenesulfonate) Films Using 1-Ethyl-3-methylimidazolium Tetracyanoborate Ionic Liquid. Adv. Funct. Mater. 2012, 22, 2723-2727. [CrossRef]

32. Lipomi, D.J.; Lee, J.A.; Vosgueritchian, M.; Tee, B.C.K.; Bolander, J.A.; Bao, Z. Electronic properties of transparent conductive films of PEDOT:PSS on stretchable substrates. Chem. Mater. 2012, 24, 373. [CrossRef]

33. Yeo, J.S.; Yun, J.M.; Kim, D.Y.; Park, S.; Kim, S.S.; Yoon, M.H.; Kim, T.W.; Na, S.I. Significant Vertical Phase Separation in SolventVapor-Annealed Poly(3,4-ethylenedioxythiophene): Poly(styrene sulfonate) Composite Films Leading to Better Conductivity and Work Function for High-Performance Indium Tin Oxide-Free Optoelectronics. ACS Appl. Mater. Interface 2012, 4, 2551. [CrossRef]

34. Xia, Y.; Ouyang, J. Significant Conductivity Enhancement of Conductive Poly(3,4-ethylenedioxythiophene): Poly(styrenesulfonate) Films through a Treatment with Organic Carboxylic Acids and Inorganic Acids. ACS Appl. Mater. Interface 2010, 2, 474. [CrossRef]

35. Ouyang, J. "Secondary doping" methods to significantly enhance the conductivity of PEDOT:PSS for its application as transparent electrode of optoelectronic devices. Displays 2013, 34, 423-436. [CrossRef]

36. Donoval, M.; Micjan, M.; Novota, M.; Nevrela, J.; Kovacova, S.; Pavuk, M.; Juhasz, P.; Jagelka, M.; Kovac Jr, J.; Jakabovic, J.; et al. Relation between secondary doping and phase separation in PEDOT:PSS films. Appl. Sur. Sci. 2017, 395, 86-91. [CrossRef] 
37. Lang, U.; Muller, E.; Naujoks, N.; Dual, J. Microscopical Investigations of PEDOT:PSS Thin Films. Adv. Func. Mater. 2009, 19, 1215-1220. [CrossRef]

38. Alemu, D.; Wei, H.Y.; Ho, K.C.; Chu, C.W. Highly conductive PEDOT:PSS electrode by simple film treatment with methanol for ITO-free polymer solar cells. Energy Environ. Sci. 2012, 5, 9662-9671. [CrossRef]

39. Park, M.U.; Shin, C.Y.; Kim, H.J.; Kim, S.Y.; Choi, Y.J.; Chung, D. Improved Coating of PEDOT:PSS onto CVD Graphene by the Addition of PVA. Appl. Chem. Eng. 2018, 29, 734-739.

40. Hwang, B.; Lim, S. PEDOT:PSS Overcoating Layer for Mechanically and Chemically Stable Ag Nanowire Flexible Transparent Electrode. J. Nanomater. 2017, 1489186. [CrossRef]

41. Park, M.U.; Song, M.; Lee, S.M.; Ryu, S.; Chung, D. Fabrication Process of Bilayer RGO/PEDOT:PSS Film for Flexible Transparent Conductive Electrode. J. Nanosci. Nanotechnol. 2018, 18, 6147-6151. [CrossRef] [PubMed]

42. Jeadd, A.; Halik, M. Toward strain resistant flexible organic thin film transistors. Appl. Phys. Lett. 2009, 95, 103309. 